\title{
Near-IR variability properties of a selected sample of AGB stars ${ }^{\star}, \star \star$
}

\author{
F. M. Jiménez-Esteban ${ }^{1,2,3}$, P. García-Lario ${ }^{4}$, D. Engels ${ }^{3}$, and A. Manchado ${ }^{5,6}$
}

1 Observatorio Astronómico Nacional, Apartado 112, 28803 Alcalá de Henares, Spain e-mail: $f$.jimenez-esteban@oan.es

2 FRACTAL SLNE, Avda San Sebastián 5 3-D, 38003 Santa Cruz de Tenerife, Spain

3 Hamburger Sternwarte, Gojenbergsweg 112, 21029 Hamburg, Germany

${ }^{4}$ Research and Scientific Support Department of ESA, European Space Astronomy Centre, Villafranca del Castillo, Apartado de Correos 50727, 28080 Madrid, Spain

5 Instituto de Astrofísica de Canarias, Via Lactea s/n, 38200 La Laguna, Tenerife, Spain

${ }^{6}$ Consejo Superior de Investigaciones Científicas, Spain

Received 4 August 2005 / Accepted 8 June 2006

\section{ABSTRACT}

\begin{abstract}
We present the results of a near-infrared monitoring programme of a selected sample of stars, initially suspected to be Mira variables and $\mathrm{OH} / \mathrm{IR}$ stars, covering more than a decade of observations. The objects monitored cover the typical range of IRAS colours shown by O-rich stars on the asymptotic giant branch and show a surprisingly large diversity of variability properties. Sixteen objects are confirmed as large-amplitude variables. Periods between 360 and $1800^{\mathrm{d}}$ and typical amplitudes $1^{\mathrm{m}} \lesssim \Delta K \lesssim 2^{\mathrm{m}}$ could be determined for nine of them. In three light curves, we find a systematic decrease in the mean brightness, and two light curves show pronounced asymmetry. One source, IRAS 07222-2005, shows infrared colours typical of Mira variables, but it pulsates with a much longer period $\left(\approx 1200^{\mathrm{d}}\right)$ than a normal Mira. Two objects are either close to (IRAS 03293+6010) or probably in (IRAS 18299-1705) the post-AGB phase. In IRAS 16029-3041 we found a systematic increase in the $H-K$ colour of $\approx 1^{\mathrm{m}}$, which we interpret as evidence of a recent episode of enhanced mass loss. IRAS 18576+0341, a heavily obscured luminous blue variable was also monitored. The star showed a continued decrease in brightness over a period of 7 years (1995-2002).
\end{abstract}

Key words. stars: AGB and post-AGB - stars: late-type - stars: variables: general - infrared: stars

\section{Introduction}

During the evolution on the asymptotic giant branch (AGB) stars begin to pulsate and appear as large-amplitude long-period variables (LALPV) during the final phase. Well-known objects are the Mira variables with optical amplitudes $>2.5^{\mathrm{m}}$ and typical periods $<400^{\mathrm{d}}$. Less known are the AGB variables that are heavily obscured in the optical. The periods of these "OH/IR stars" are considerably longer than those of optical Mira variables. Observing in the infrared, Engels et al. (1983) derived the periods of 17 objects they monitored, finding values between $500^{\mathrm{d}}$ and $1800^{\mathrm{d}}$ and very large amplitudes (up to $\approx 4^{\mathrm{m}}$ ) in the $K$ band. Herman \& Habing (1985) found similar periods from the monitoring of the $\mathrm{OH}$ maser emission. The upper limits of the period distribution on the AGB could not be determined, however, as the monitor projects were not continued long enough.

We started a new open-end infrared monitoring programme in 1991 making use of the service time option at the Observatorio del Teide. The aim was to determine variability properties of a sample of AGB stars with a wide range of mid-infrared IRAS colours, including some stars suspected of having extremely long periods. Although in the meantime various authors (Nakashima et al. 2000; Blommaert et al. 1998; Wood et al. 1998; Wood 1998; Jones et al. 1994; van Langevelde et al. 1993, 1990; Le Bertre 1993) have provided periods for

* Table A.1 is only available in electronic form at the CDS via anonymous ftp to cdsarc.u-strasbg.fr $(130.79 .128 .5)$ or via http://cdsweb.u-strasbg.fr/cgi-bin/qcat?J/A+A/458/533

$\star \star$ Figures A.1 and A.2 are only available in electronic form at http://www. aanda.org additional $\mathrm{OH} / \mathrm{IR}$ stars, the number of $\mathrm{OH} / \mathrm{IR}$ stars studied for variability is still low compared to the number of Mira variables monitored.

In this paper we present the observations of 24 sources suspected of being AGB stars and obtained between 1991 and 2002. Due to the service time character of the programme, the observations were made irregularly so their number is sometimes very limited. Nevertheless, we are able to provide a handful of new period and amplitude determinations of Miras and OH/IR stars.

\section{Sample selection}

The sample contains 24 stars selected from the IRAS Point Source Catalogue with well-measured fluxes (FQUAL $\geq 2$, Beichman et al. 1988) in the IRAS photometric bands centred at 12,25 , and $60 \mu \mathrm{m}$ and with colours that resemble those typically shown by O-rich AGB stars. The selection was made in 1990 and none of the stars had been studied before. All objects monitored are listed in Table 1. The table gives the IRAS name, the [12]-[25] IRAS colour, the J2000 equatorial coordinates, and several columns with the results of the variability analysis, which are described in Sect. 5. All sources but IRAS 02095-2355, IRAS $04130+3918$, IRAS $18576+0341$, and IRAS 23492+0846 were previously detected as $\mathrm{OH}$ masers at $1612 \mathrm{MHz}$ (te Lintel Hekkert et al. 1989, 1991; Zijlstra et al. 1989; David et al. 1993), confirming their oxygen-rich chemistry. IRAS 02095-2355 and IRAS 23492+0846 turned out to be M-giants (Whitelock et al. 1995), while IRAS $04130+3918$ has recently been identified as a carbon star (Alksnis et al. 2001) and IRAS 18576+0341 as a luminous blue variable star (Clark et al. 2003). 

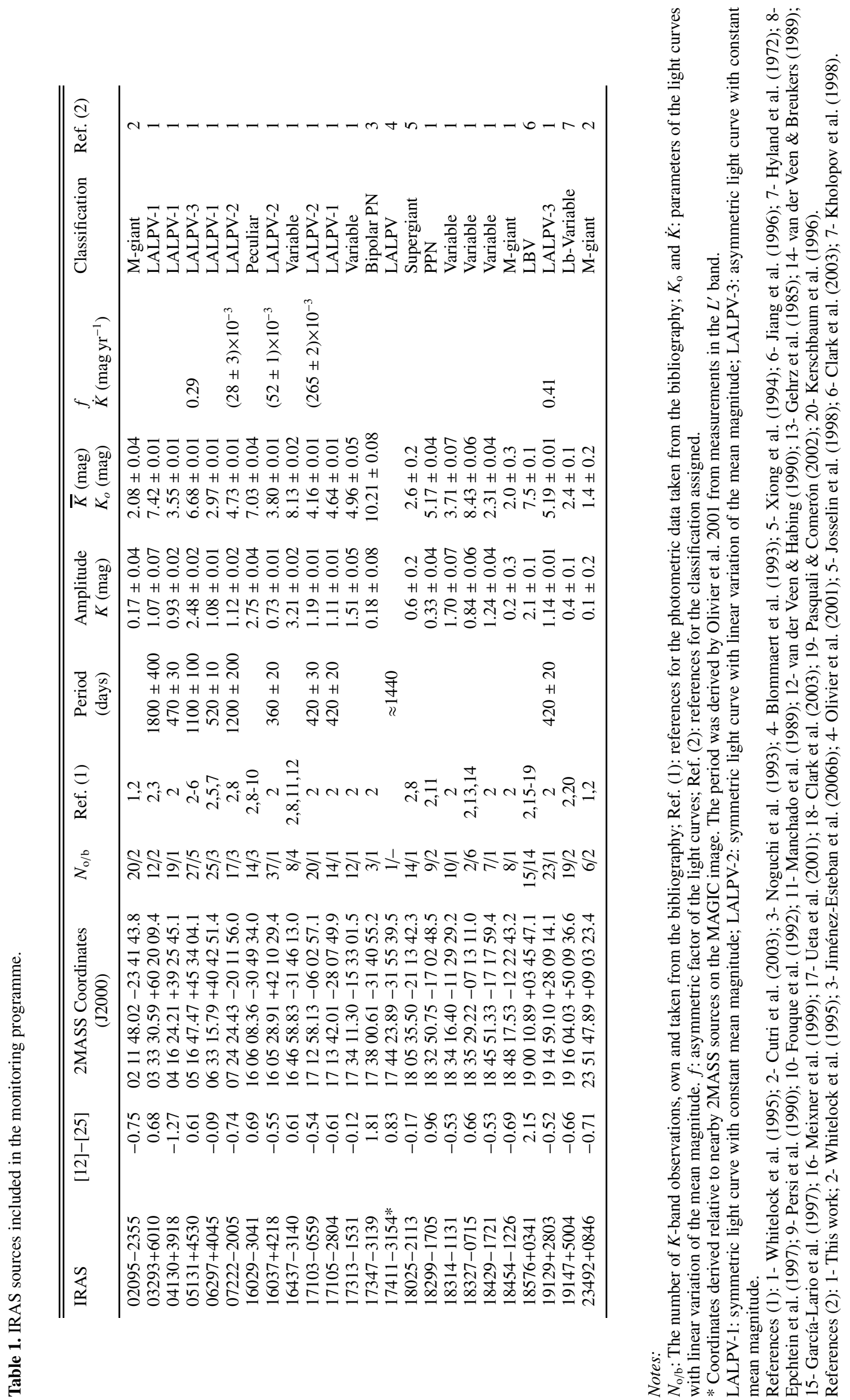


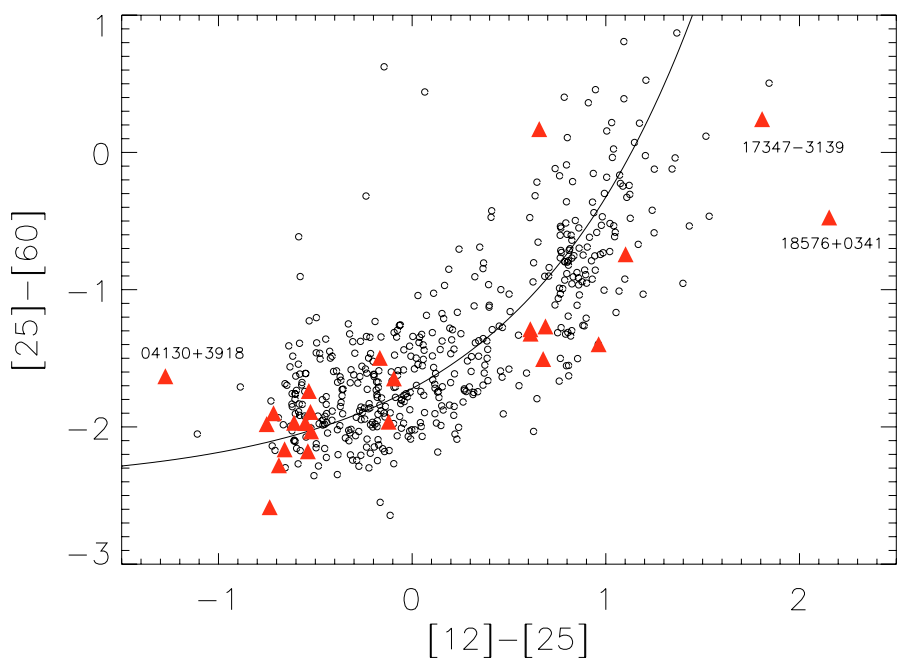

Fig. 1. IRAS colour-colour diagram showing the location of the stars included in the monitoring programme (filled triangles), together with oxygen-rich AGB stars from the "Arecibo sample" (JE05) and from the "GLMP sample" (JE06) (open circles). The continuous line is the "O-rich AGB sequence", and the IRAS colours are defined as: $[12]-[25]=-2.5 \log \frac{F_{v}(12)}{F_{v}(25)}$ and $[25]-[60]=-2.5 \log \frac{F_{v}(25)}{F_{v}(60)}$.

In Fig. 1 we show the location of the sample in an IRAS colour-colour diagram. The sources were intentionally selected to be located at different positions along the "O-rich AGB sequence", as is defined by the oxygen-rich AGB star samples of Jiménez-Esteban et al. (2005, 2006a; hearafter JE05, JE06). Two exceptions are IRAS 17347-3139 and IRAS 18576+0341, which are placed in the region corresponding to sources with detached shells (like post-AGB stars or planetary nebulae). The Mira variables populate the blue and the $\mathrm{OH} / \mathrm{IR}$ stars the red part. It was therefore expected to find Mira-like variability with increasing periods and amplitudes along the "O-rich AGB sequence".

\section{Observations}

The observations presented here were carried out over a period of 10 years, from 1991 March to 2002 August. During the first years we performed aperture photometry, mainly using service time at the infrared $1.5 \mathrm{~m}$ Carlos Sánchez Telescope (CST) of the Observatorio del Teide (Canary Islands, Spain). Later, we complemented the monitoring programme with infrared images taken at the $1.23 \mathrm{~m}$ telescope at Calar Alto Observatory (Almería, Spain).

\subsection{Aperture photometry}

Aperture photometry at the $1.5 \mathrm{~m} \mathrm{CST}$ was performed with a Circular Variable Filter (CVF) spectrophotometer. The CVF is equipped with an InSb photometric detector, which works at the temperature of liquid nitrogen. A photometric aperture of $15^{\prime \prime}$ with a chopper throw of $30^{\prime \prime}$ in right ascension was used to subtract the contribution from the background sky. We used the infrared filters $J(1.25 \mu \mathrm{m}), H(1.65 \mu \mathrm{m}), K(2.20 \mu \mathrm{m})$, and occasionally $L^{\prime}(3.8 \mu \mathrm{m})$. The Teide photometric system and its transformation to other standard photometric systems are described in Arribas \& Roger (1987). All observations were taken under photometric weather conditions. For the photometric calibration, we used the list of standard stars in Koornneef (1983), observing at least two of these several times every night at different air masses to obtain the extinction coefficients. The service observations were solicited imposing a minimum $S / N$ ratio of 50 in the $K$ band. All magnitudes given in this paper are in the Teide photometric system.

\subsection{Imaging photometry}

In the final years of the monitoring programme we also used the $1.23 \mathrm{~m}$ telescope of the Calar Alto Observatory in three different runs, equipped with the infrared camera MAGIC (Herbst et al. 1993). Observations were made in the broad-band filters $J, H$, and $K$. The camera has a $256 \times 256$ NICMOS $3 \mathrm{HgCdTe}$ detector array with a pixel scale of $1.2^{\prime \prime}$, which provides an approximate field of view of $5^{\prime} \times 5^{\prime}$. Several standard stars from the list of Elias et al. (1982) were used for the calibration of near-infrared images taken under photometric weather conditions. Images taken during non-photometric nights were calibrated using 2MASS Point Source Catalogue (Cutri et al. 2003). The observational technique and the data reduction process are described in detail in JE05.

We found no differences in magnitudes and colours between the Calar Alto photometric system and the "CIT" system used by Elias et al. (1982). To transform the Calar Alto photometry (CA) to the Teide photometric system (OT), we used the transformations given by Arribas \& Roger (1987), which relate the Teide to the "CIT" system. Actually, only the $J$-band photometry is affected: $K_{\mathrm{OT}}=K_{\mathrm{CIT}}=K_{\mathrm{CA}}, H_{\mathrm{OT}}=H_{\mathrm{CIT}}=H_{\mathrm{CA}}$, and $(J-H)_{\mathrm{OT}}=1.11(J-H)_{\mathrm{CIT}}=1.11(J-H)_{\mathrm{CA}}$.

\section{Near-infrared photometry}

The near-infrared counterparts of the IRAS sources were initially searched for with the CVF spectrophotometer by scanning in the vicinity of the IRAS coordinates, which have typical errors $20^{\prime \prime}-30^{\prime \prime}$. In most cases only one source showing colours typical of AGB stars was found. This source actually was also the reddest in the field and, as such, easily recognizable as the right counterpart.

The counterparts were later verified by using the MAGIC images from the Calar Alto observations and by checking the spectral energy distributions with the help of the MSX Point Source Catalogue (Egan et al. 2003) and the 2MASS Point Source Catalogue. Except for IRAS 17411-3154, the original counterparts could be confirmed. The best available positions as given by the 2MASS are listed in Table 1.

In the case of IRAS 17411-3154, we found the counterpart to be strongly blended by a foreground star and to be detectable only during the bright phases of the light variation cycle and only in the $K$-band (see Fig. 2). The object was monitored in parallel by Olivier et al. (2001) at $3.45 \mu \mathrm{m}$, and turned out to be a LALPV with a period of $P=1440^{\mathrm{d}}$. The coordinates given in Table 1 for this source were derived relative to the 2MASS positions of nearby sources in the field.

The near-infrared $J H K L^{\prime}$ photometric data, together with their associated errors, are listed in Table A.1 of the Appendix. Although the data has been collected during 121 observing nights, the number of observations per object was much smaller and rather inhomogeneous. A minimum of six and a maximum of 37 observations were accumulated for individual objects. The errors obtained for the aperture photometry are in the range $0.01-0.1^{\mathrm{m}}$ for the majority of cases, while those obtained for imaging photometry are typically $\approx 0.03^{\mathrm{m}}$.

Judging from the MAGIC infrared images, we found a number of cases where the infrared fluxes obtained with the CVF 

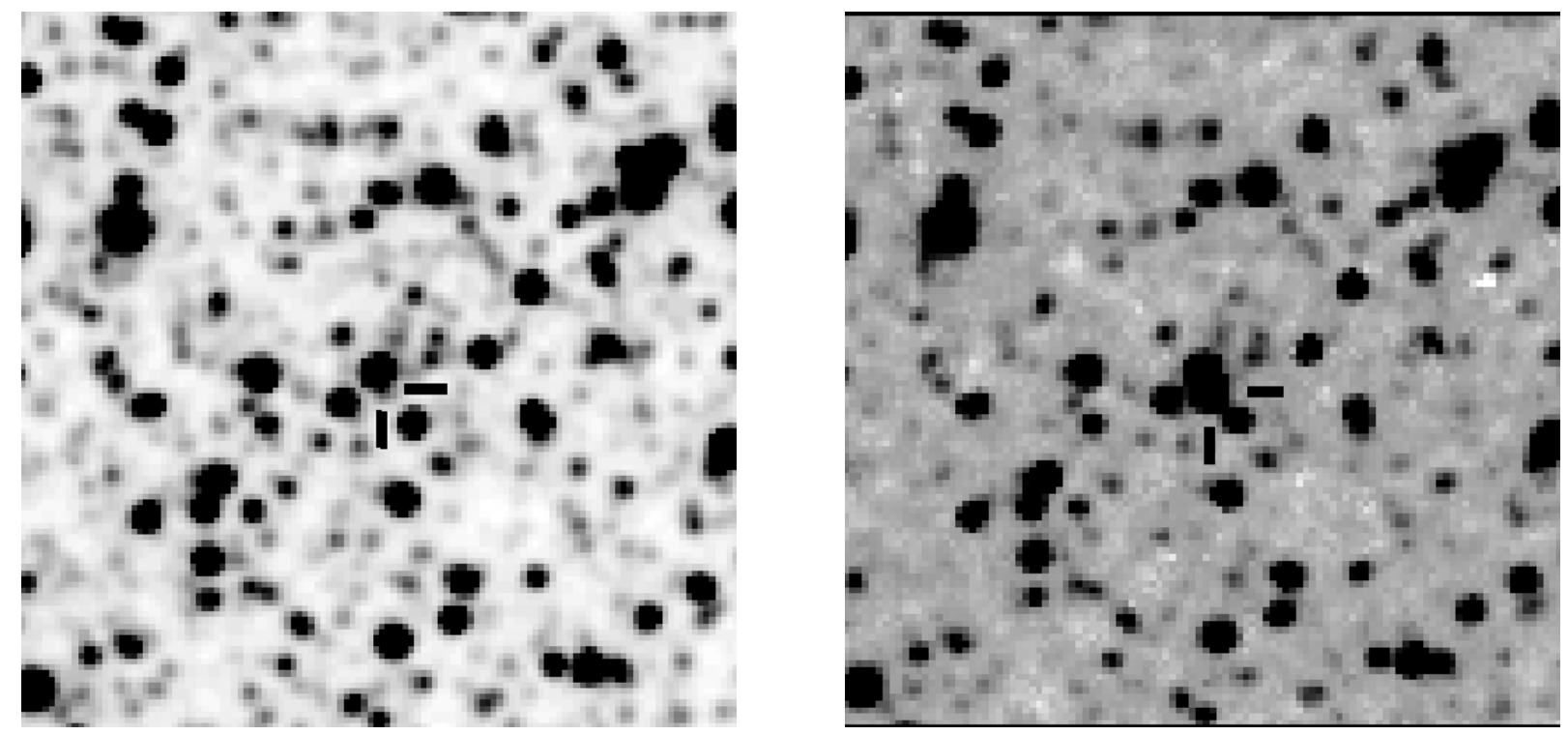

Fig. 2. $K$-band images of IRAS 17411-3154 in two different epochs: 1998 (2MASS; left panel) and 2001 (MAGIC; right panel).

spectrophotometer must have been contaminated by nearby stars contained in the aperture. IRAS 16437-3140, for instance, was strongly blended with an object having similar brightness in the $J$-band, but much fainter in the $H$ and $K$ bands. We therefore removed the photometry in the $J$ filter for this source from Table A.1. Other sources affected were IRAS 17347-3139 and IRAS 17411-3154, where none of the aperture photometry data turned out to be useful.

As the light variations were rather similar in all three bands, we present graphically here only light curves from the $K$-band data, which is the most complete data set. They are plotted in Fig. A.1 of the Appendix, excluding IRAS 17347-3139 and IRAS 17411-3154. We added $K$ band photometry from the 2MASS and DENIS (Epchtein et al. 1997) near-infrared surveys, and from the literature (Hyland et al. 1972; Manchado et al. 1989; Persi et al. 1990; van der Veen \& Habing 1990; Blommaert et al. 1993; Noguchi et al. 1993; Xiong et al. 1994; Jiang et al. 1996). Whenever a model light curve was fitted to the observational data (see the following section) this is also shown. The number of $K$-band observations $\left(N_{\mathrm{o} / \mathrm{b}}=\right.$ own and contributed $)$ used is given in Table 1.

\section{Light curve analysis}

We characterize the variability of the sources on the basis of the amplitudes and periods listed in Table 1. If periods are given, the light curves were successfully fitted by a periodic model light curve (see next section) and the amplitudes are those derived from the fit. In the other case, amplitudes are taken as the difference between the observed maximum and minimum brightness.

Conclusions on variability can be made for 23 of the 24 sources of Table 1 . We found variability with amplitudes $\Delta K>0.2$ for all sources except IRAS 02095-2355, IRAS 17347-3139, IRAS 18454-1226, and IRAS 23492+0846. The latest sources only show some scattering and can be considered as essentially non-variable. IRAS $17347-3139$ is a bipolar planetary nebula (Jiménez-Esteban et al. 2006b). The three other stars are rather bright $\left(K<2.1^{\mathrm{m}}\right)$ and belong to the bluest sources in the sample $([12]-[25]<-0.65)$. Two of them were classified as non-Mira M-giants because of their near-infrared colours by Whitelock et al. (1995). This classification is corroborated by their non-variability. For the same reasons, we identified the third source IRAS 18454-1226 with similar infrared and variability properties as M-giant as well. The classifications adopted and their origin are listed in the last two columns of Table 1.

The variable sources can be split into a group of regularly pulsating large-amplitude long-period variables (LALPVs) and a group with ambiguous variability properties.

In order to characterize the variability properties of the LALPVs, we fitted model light curves of several types to the observational data, including data from the literature. As a first approach, we adopted a symmetric sinusoidal model light curve (Type 1). The least mean square (LMS) method was applied for a range of periods between 200 and $3000^{\mathrm{d}}$. The quality of the fit was determined with the $\chi^{2}$ test, weighting each observation with the inverse of the square of the observational error.

We checked our results using the period dispersion minimization (PDM) method (Stellingwerf 1978). The errors $\Delta P$ for the period estimations were derived according to the PDM method from $\Delta P \simeq P^{2} / 2 T$, where $P$ is the trial period at the primary minimum, and $T$ is the time baseline of the observations.

We found that in some cases assuming a symmetric sinusoidal curve and a constant mean magnitude is not the best choice. Some objects, like IRAS 17103-0559 (See Fig. A.1), show clear evidence of large secular variations in their nearinfrared mean brightness. We therefore also used symmetric sinusoidal model light curves with a mean magnitude linearly variable with time (type 2). This type of light curve was adopted, if we obtained a value of $\chi^{2}$ that is at least $30 \%$ smaller than the one derived from fitting type 1 light curve.

The third type of model light curve assumed a constant mean magnitude but an asymmetric sine (type 3 ) characterized by an asymmetry factor $f$. This factor can assume values in the range 0 and 1 , with $f=0.5$ corresponding to a symmetric light curve. A value $<0.5(>0.5)$ corresponds to a steeper (shallower) rising branch compared to the descending branch (van Langevelde et al. 1993). A good case for an asymmetric sinusoidal light curve is given by IRAS 05131+4530 (see Fig. A.1). For this source a similar period was previously reported by 


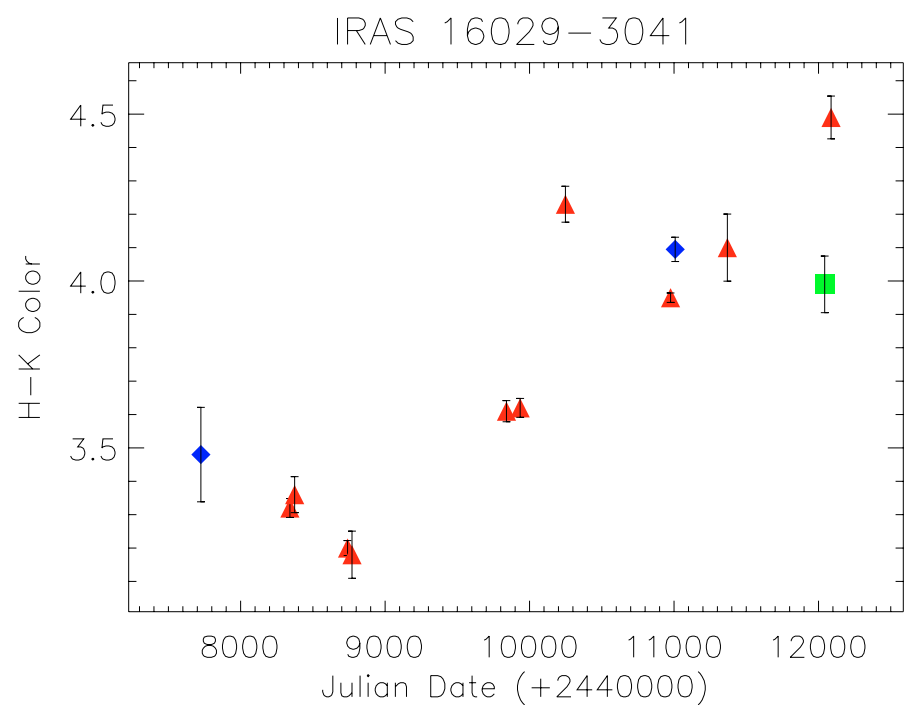

Fig. 3. Variation of the $H-K$ colour with time for IRAS 16029-3041. CVF data are plotted with triangles (red), MAGIC data with squares (green), and data from literature with diamonds (blue).

Groenewegen et al. (1999). As before, this type was only adopted if $\chi^{2}$ was found to be at least $30 \%$ smaller than its value for type 1 or type 2 light curves.

For nine sources we successfully fitted model light curves. They were classified as LALPV-n in Table 1 , where $n=1,2,3$ depend on the type of light curve adopted. The light curves are shown in Figs. A.1 and A.2 of the Appendix, as functions of time and phase. Their periods, amplitudes, and corresponding errors are given in Table 1 . In addition, the mean magnitudes $\bar{K}$ are given for light curves of type 1, the slopes of the mean magnitude $\dot{K}$ and the mean magnitudes $K_{\mathrm{o}}$ at Julian Date 2450000 are listed for type 2 light curves, and $\bar{K}$ and the asymmetry factors $f$ are given for type 3 light curves. Periods between $360^{\mathrm{d}}$, typical of Mira variables, and $1800^{\mathrm{d}}$ were derived. The long periods of $>1000^{\mathrm{d}}$ obtained for three stars are in the range of OH/IR star periods found in earlier studies.

The objects with ambiguous variability properties consist of a group of stars with irregular variability behaviour and others that probably have an insufficient amount of observations to determine the periodicity of their variations.

Irregular low-amplitude variability $\left(\Delta K \leq 0.6^{\mathrm{m}}\right)$ is shown by IRAS 18025-2113, IRAS 18299-1705, and IRAS 19147+5004 (TZCyg). IRAS 18025-2113, a possible supergiant star (Josselin et al. 1998), and IRAS 19147+5004, an Lb-type irregular variable (Kholopov et al. 1998), are bright $\left(\bar{K}<3^{\mathrm{m}}\right)$ and blue in the mid-infrared $([12]-[25]<-0.1)$. Their presence indicates that a fraction of the stars populating the blue end of the "O-rich AGB sequence" are actually not genuine Mira variables.

In contrast, the third low-amplitude variable IRAS $18299-1705$ is fainter $(\bar{K}=5.2)$ and has one of the reddest [12]-[25] colours in the sample. The low amplitude indicates that this star belongs to the group of "non-variable" $\mathrm{OH} / \mathrm{IR}$ stars. This group shows irregular low-amplitude variability (if any) in contrast to the regular pulsating OH/IR stars with large amplitudes. Because of the cessation of large-amplitude pulsations, they are considered as stars that are leaving the AGB and in which the strong mass-loss has finished (Engels 2002). In the case of IRAS 18299-1705, the circumstellar shell has been diluted so far that the central star has reappeared again and can be detected in the near-infrared. Therefore, we tentatively classify this star as a proto-planetary nebula. Note, however, that in principle similar observational properties can also be associated to AGB stars during the helium-burning cycle following a thermal pulse, when a sharp decrease in the mass-loss rate is also expected, leading to the development of a detached dust shell.

A peculiar variability behaviour is shown by IRAS 160293041 and IRAS $18576+0341$. IRAS 16029-3041 shows strong variability with one of the largest amplitudes $\left(\Delta K \approx 2.75^{\mathrm{m}}\right)$ in our sample. This object actually shows the reddest near-infrared colours, but these colours have been changing during the years of monitoring. In Fig. 3 the evolution of its $H-K$ colour as a function of the Julian Date is shown, which has increased by $\approx 1^{\mathrm{m}}$ in the past 10 years. This change in the near-infrared colour is accompanied by a simultaneous fading in the $K$ and $H$ bands, as might be expected in case of high, increasing extinction. This object must have a very thick CSE as derived from its lack of an optical counterpart, which suggests a large mass-loss experienced in the recent past. The progressive reddening is interpreted as the result of a continuous increase in the thickness of its CSE, which has yet not finished, probably due to a very recent episode of enhanced mass loss.

A special case is also IRAS $18576+0341$. This star is not on the AGB, but is a luminous blue variable star (Clark et al. 2003). It has a rather slowly varying light curve, which reached a maximum at $K=6.5^{\mathrm{m}}$ in 1995 and descended since then by $\approx 1.5^{\mathrm{m}}$ until the last available measurement by the end of 2002 .

The remaining five stars, classified as "variable" in Table 1 , show a wide range of amplitudes $\left(0.8^{\mathrm{m}}<\Delta \mathrm{K}<3.3^{\mathrm{m}}\right)$, but have a relatively small number of observations $(N \lesssim 15)$. For some of them we could fit a periodic model light curve, however the significance was low. For others, irregular variations look more likely. Additional photometric data points taken with a higher observing frequency are required to classify the light curves of these variables correctly.

\section{Discussion}

The monitoring programme confirmed that the large majority of the IRAS sources along the 'O-rich AGB sequence' are indeed variable. Non-variable M-giants are part of the population only at the blue end of the sequence. One of them, IRAS 18454-1226, was detected as an OH maser (te Lintel Hekkert et al. 1991), implying that non-pulsating M-giants may also possess a circumstellar gas and dust shell able to support $\mathrm{OH}$ emission.

Variability has been found on all amplitude scales. Lowamplitude variability ( $\Delta K \leq 0.6 \mathrm{mag})$ has been found for two stars among the blue sources that can be characterized as irregular variables. Irregular, low-amplitude variations were also found for the very red source IRAS 18299-1705. Together with the presence of a rather bright near-infrared counterpart, this star is probably already in the post-AGB stage. IRAS 182991705 shows that post-AGB stars can be found relatively close to the red end of the "O-rich AGB sequence" and that there is an overlap between the locations of heavily obscured late AGB stars pulsating with large amplitudes and the "non-variable" post-AGB stars having, in general, redder [12]-[25] colours. A similar conclusion was drawn by JE05, who discovered several bright near-infrared counterparts to very red objects on the "Orich AGB sequence".

Large-amplitude variations were found for 16 sources. Two are peculiar. One, IRAS $18576+0341$, is not an AGB star and will not be discussed further. IRAS 16029-3041 has a strong double-peaked $\mathrm{OH}$ maser and the IRAS Low Resolution 


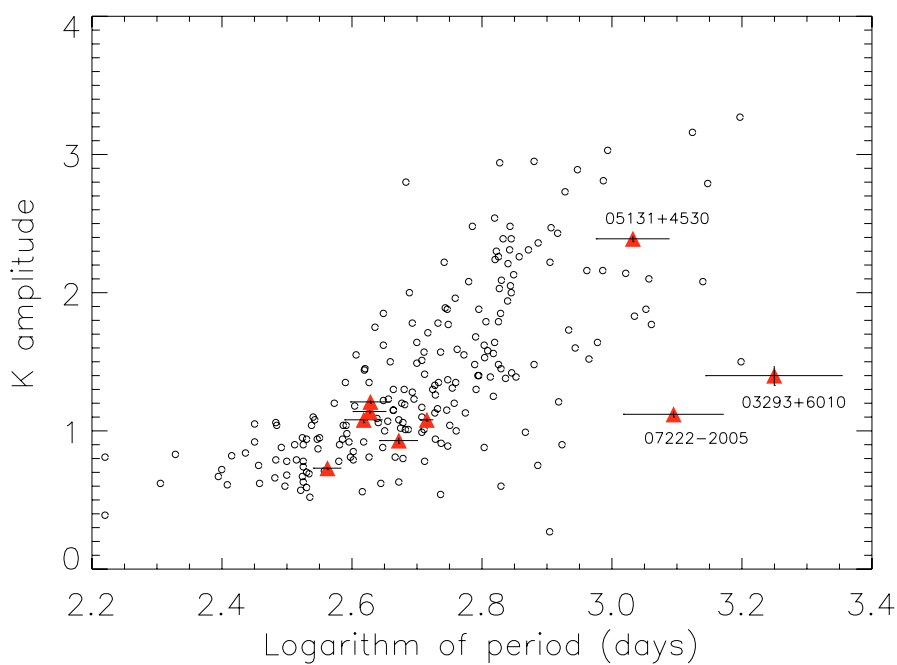

Fig. 4. $K$ amplitude $(\Delta K)$ as a function of the period. We have plotted our data (filled triangles), together with data extracted from the bibliography (open circles).

Spectrum has an index 32, characterizing oxygen-rich dust shells with the $10 \mu \mathrm{m}$ feature in absorption. It is definitely an AGB star. The erratic light curve (see Fig. A.2) connected to a systematic reddening of the near-infrared colours is suggested to be the result of a recent episode of enhanced mass loss.

The service-time character of the monitoring programme had the disadvantage that for several sources (labeled "variable" in Table 1) the observing frequency was too low to prove periodicity in their light curves. For nine sources, however, regular pulsation could be proven, and we found the expected wide range of periods between 360 and $1800^{\mathrm{d}}$, and amplitudes up to $\Delta K=2.5^{\mathrm{m}}$. Due to the long duration of the monitor programme, observations were usually spread over several pulsation cycles. We did not find significant cycle-to-cycle variations in 6 cases, but for three stars a systematic decrease of the mean near-infrared brightness was found. Such secular variations in the mean brightness have been reported in several other longperiod variables (Bedding et al. 2002; Whitelock et al. 2003). The secular variations might be connected to changes in the mass-loss rate, which lead to a varying obscuration of the star in the near-infrared.

For IRAS 05131+4530 and IRAS 19129+2803, a significant asymmetry of the light curve was found. These asymmetries are commonly observed in the optical light curves of Mira variables (Vardya 1988) but are less pronounced in the infrared.

In our sample we find stars with very similar periods, but very different amplitudes. In Fig. 4 we plotted our results with data taken from the literature (Olivier et al. 2001; Wood et al. 1998; Whitelock et al. 1994; Le Bertre 1993; Engels et al. 1983). Up to $\log P=2.55$, a small range of amplitudes $0.7^{\mathrm{m}} \leq \Delta K \leq 1.1^{\mathrm{m}}$ is observed, while the amplitudes increase steadily with longer periods. However, the scatter is large. Two sources of our sample, IRAS 03293+6010 and IRAS 07222-2005, have relatively low amplitudes for their periods. IRAS $03293+6010$ has red mid-infrared colours and the longest period $\left(P=1800^{\mathrm{d}}\right)$ in the sample. IRAS 07222-2005, in contrast, has very blue midinfrared colours and an unusually long period $\left(P=1200^{\mathrm{d}}\right)$ for this colour.

We speculate that IRAS $03293+6010$ is in an advanced evolutionary stage, in which the large-amplitude pulsation is fading due to the rapidly decreasing envelope mass, leading to very lengthened periods and decreasing amplitudes. The star might be close to the transition to the post-AGB stage and may develop currently into a "non-variable" $\mathrm{OH} / \mathrm{IR}$ star.

For IRAS 07222-2005, it is not the amplitude of its pulsation but the length of the period that is peculiar. There are no other stars known with such a long period located at the blue end of the 'O-rich AGB sequence'. The star has a strong silicate feature in emission (IRAS LRS $=29$ ) and a low gas outflow velocity of $8.1 \mathrm{~km} \mathrm{~s}^{-1}$ according to the $\mathrm{OH}$ maser (te Lintel Hekkert et al. 1991). IRAS 07222-2005 is therefore not an M-Supergiant. Similar to IRAS 03293+6010, the long period of IRAS 07222-2005 might be due to a small remaining envelope mass, while approaching the end of the AGB. Contrary to IRAS 03293+6010, however, IRAS 07222-2005 may be finishing the AGB with a modest mass-loss rate and, accordingly, with a blue colour. A reduced mass loss is expected for stars with their $\mathrm{C} / \mathrm{O}$ ratio being close to unity, thus hampering dust formation (Willems \& de Jong 1988; Chan \& Kwok 1988). Alternatively, IRAS 07222-2005 can be similar to some MCs OH/IR stars with $P>1000^{\mathrm{d}}$ and rather blue colours, like e.g. IRAS 04509-6922 with $\langle H-K\rangle=0.85, \Delta K=1.5^{\mathrm{m}}$, and $P=1290^{\mathrm{d}}$ (see Table 5 of Wood et al. 1992). They are rather luminous and therefore probably massive. The lower metallicity of the LMC compared to the Galaxy might have decreased their mass-loss rate. Interestingly, IRAS 07222-2005 is located in the anticentre direction $\left(l=234^{\circ}\right.$, $\left.b=-2^{\circ}\right)$ compared to other monitored objects which are mostly at $\left(10^{\circ}<l<45^{\circ}\right)$, and, it shows a relatively low expansion velocity $^{1}$, which is compatible with lower metallicity as in the LMC.

\section{Conclusions}

The monitoring programme has shown that the variability properties along the "O-rich AGB sequence" are diversified. In general, periods and amplitudes increase with redder colours, but the scatter is large, and AGB stars in evolutionary phases of nonor low-amplitude variability are found at the borders of the sequence. The transition to post-AGB stars in the IRAS colourcolour diagram appears to be smooth. The variability properties certainly hold important clues to the evolutionary stage of the stars on the AGB. To uncover the links between these properties and particular phases of AGB evolution requires, however, larger samples and therefore long-term monitoring programmes to study the variability properties, especially at the red end of the "O-rich AGB sequence", should be encouraged.

Acknowledgements. Based on service-time observations made with the Carlos Sánchez telescope of the Observatorio del Teide (Tenerife, Spain), operated by the Instituto de Astrofísica de Canarias, and on observations collected at the German-Spanish Astronomical Centre, Calar Alto, operated jointly by the Max-Planck-Institut für Astronomie and the Instituto de Astrofísica de Andalucía (CSIC). This work was partially funded through grants PB94-1274 from the Spanish Dirección General de Investigación Científica y Técnica (DGICYT) and AYA2003-06473, AYA2003-09499, and AYA2004-03136 from the Spanish Ministerio de Ciencia y Tecnología. This publication makes use of data products from the Two Micron All Sky Survey, which is a joint project of the University of Massachusetts and the Infrared Processing and Analysis Center/California Institute of Technology, funded by the National Aeronautics and Space Administration and the National Science Foundation. We also thank to the referee for valuable suggestions.

\footnotetext{
1 The expansion velocity is derived from a questionable $\mathrm{OH}$ $1612 \mathrm{MHz}$ detection by te Lintel Hekkert (1990). The measurement is not listed in Table 2 of te Lintel Hekkert et al. (1991) (printed version), but is contained in the electronic version of the table. The maser and derived velocities therefore require confirmation (te Lintel Hekkert, priv. communication).
} 


\section{References}

Alksnis, A., Balklavs, A., Dzervitis, U., et al. 2001, Balt. Astron., 10, 1

Arribas, S., \& Roger, C. M. 1987, A\&AS, 70, 303

Bedding, T. R., Zijlstra, A. A., Jones, A., et al. 2002, MNRAS, 337, 79

Beichman, C. A., Neugebauer, G., Habing, H. J., Clegg, P. E., \& Chester, T. J. 1988, in Infrared astronomical satellite (IRAS) catalogs and atlases. Vol. 1: Explanatory supplement

Blommaert, J. A. D. L., van der Veen, W. E. C. J., \& Habing, H. J. 1993, A\&A, 267, 39

Blommaert, J. A. D. L., van der Veen, W. E. C. J., van Langevelde, H. J., Habing, H. J., \& Sjouwerman, L. O. 1998, A\&A, 329, 991

Chan, S. J., \& Kwok, S. 1988, ApJ, 334, 362

Clark, J. S., Larionov, V. M., Crowther, P. A., Egan, M. P., \& Arkharov, A. 2003, A\&A, 403, 653

Cutri, R. M., Skrutskie, M. F., van Dyk, S., et al. 2003, in Two Micron All Sky Survey (2MASS) All-Sky Catalog of Point Sources

David, P., Lesqueren, A. M., \& Sivagnanam, P. 1993, A\&A, 277, 453

Egan, M. P., Price, S. D., \& Kraemer, K. E. 2003, in The Midcourse Space Experiment (MSX) Point Source Catalog Version 2.3

Elias, J. H., Frogel, J. A., Matthews, K., \& Neugebauer, G. 1982, AJ, 87, 1029

Engels, D. 2002, A\&A, 388, 252

Engels, D., Kreysa, E., Schultz, G. V., \& Sherwood, W. A. 1983, A\&A, 124, 123

Epchtein, N., de Batz, B., Capoani, L., et al. 1997, The Messenger, 87, 27

Fouque, P., Le Bertre, T., Epchtein, N., Guglielmo, F., \& Kerschbaum, F. 1992, A\&AS, 93, 151

García-Lario, P., Manchado, A., Pych, W., \& Pottasch, S. R. 1997, A\&AS, 126, 479

Gehrz, R. D., Hackwell, J. A., Grasdalen, G. L., Kleinmann, S. G., \& Mason, S. 1985, ApJ, 290, 296

Groenewegen, M. A. T., Baas, F., Blommaert, J. A. D. L., et al. 1999, A\&AS, 140, 197

Herbst, T. M., Beckwith, S. V., Birk, C., et al. 1993, in Proc. SPIE Vol. 1946, p. 605-609, Infrared Detectors and Instrumentation, ed. Albert M. Fowler, 605-609

Herman, J., \& Habing, H. J. 1985, A\&AS, 59, 523

Hyland, A. R., Becklin, E. E., Frogel, J. A., \& Neugebauer, G. 1972, A\&A, 16, 204

Jiang, B. W., Deguchi, S., \& Nakada, Y. 1996, AJ, 111, 231

Jiménez-Esteban, F. M., Agudo-Mérida, L., Engels, D., \& García-Lario, P. 2005, A\&A, 431, 779

Jiménez-Esteban, F. M., García-Lario, P., Engels, D., \& Perea Calderón, J. V. 2006a, A\&A, 446, 773
Jiménez-Esteban, F. M., Perea Calderón, J. V., Suárez, O., Bobrowsky, M., \& García-Lario, P., 2006b, Proc. IAU Symp. 234, Planetary Nebulae in Our Galaxy and Beyond (3-7 Apr. 2006), eds. M. J. Barlow \& R. H. Mendez (Cambridge Univ. Press), in press [arXiv: astro-ph/0608290]

Jones, T. J., McGregor, P. J., Gehrz, R. D., \& Lawrence, G. F. 1994, AJ, 107, 1111

Josselin, E., Loup, C., Omont, A., et al. 1998, A\&AS, 129, 45

Kerschbaum, F., Lazaro, C., \& Habison, P. 1996, A\&AS, 118, 397

Kholopov, P. N., Samus, N. N., Frolov, M. S., et al. 1998, in Combined General Catalogue of Variable Stars, 4.1 Ed (II/214A)

Koornneef, J. 1983, A\&AS, 51, 489

Le Bertre, T. 1993, A\&AS, 97, 729

Manchado, A., Garcia-Lario, P., Esteban, C., Mampaso, A., \& Pottasch, S. R. 1989, A\&A, 214, 139

Meixner, M., Young Owl, R., \& Leach, R. W. 1999, PASP, 111, 997

Nakashima, J., Jiang, B. W., Deguchi, S., Sadakane, K., \& Nakada, Y. 2000, PASJ, 52, 275

Noguchi, K., Qian, Z., Wang, G., \& Wang, J. 1993, PASJ, 45, 65

Olivier, E. A., Whitelock, P., \& Marang, F. 2001, MNRAS, 326, 490

Pasquali, A. \& Comerón, F. 2002, A\&A, 382, 1005

Persi, P., Ferrari-Toniolo, M., Ranieri, M., Marenzi, A., \& Shivanandan, K. 1990, A\&A, 237, 153

Stellingwerf, R. F. 1978, ApJ, 224, 953

te Lintel Hekkert, P. 1990, Ph.D. Thesis

te Lintel Hekkert, P., Versteege-Hensel, H. A., Habing, H. J., \& Wiertz, M. 1989, A\&AS, 78, 399

te Lintel Hekkert, P., Caswell, J. L., Habing, H. J., et al. 1991, A\&AS, 90, 327

Ueta, T., Meixner, M., Dayal, A., et al. 2001, ApJ, 548, 1020

van der Veen, W. E. C. J., \& Breukers, R. J. L. H. 1989, A\&A, 213, 133

van der Veen, W. E. C. J., \& Habing, H. J. 1990, A\&A, 231, 404

van Langevelde, H. J., Janssens, A. M., Goss, W. M., Habing, H. J., \& Winnberg, A. 1993, A\&AS, 101, 109

van Langevelde, H. J., van der Heiden, R., \& van Schooneveld, C. 1990, A\&A, 239,193

Vardya, M. S. 1988, A\&AS, 73, 181

Whitelock, P., Menzies, J., Feast, M., et al. 1994, MNRAS, 267, 711

Whitelock, P., Menzies, J., Feast, M., et al. 1995, MNRAS, 276, 219

Whitelock, P. A., Feast, M. W., van Loon, J. T., \& Zijlstra, A. A. 2003, MNRAS, 342, 86

Willems, F. J., \& de Jong, T. 1988, A\&A, 196, 173

Wood, P. R. 1998, A\&A, 338, 592

Wood, P. R., Whiteoak, J. B., Hughes, S. M. G., et al. 1992, ApJ, 397, 552

Wood, P. R., Habing, H. J., \& McGregor, P. J. 1998, A\&A, 336, 925

Xiong, G. Z., Chen, P. S., \& Gao, H. 1994, A\&AS, 108, 661

Zijlstra, A. A., Te Lintel Hekkert, P., Pottasch, S. R., et al. 1989, A\&A, 217, 157 
F. M. Jiménez-Esteban et al.: Near-IR variability properties of a selected sample of AGB stars, Online Material p 1

\section{Online Material}


F. M. Jiménez-Esteban et al.: Near-IR variability properties of a selected sample of AGB stars, Online Material p 2
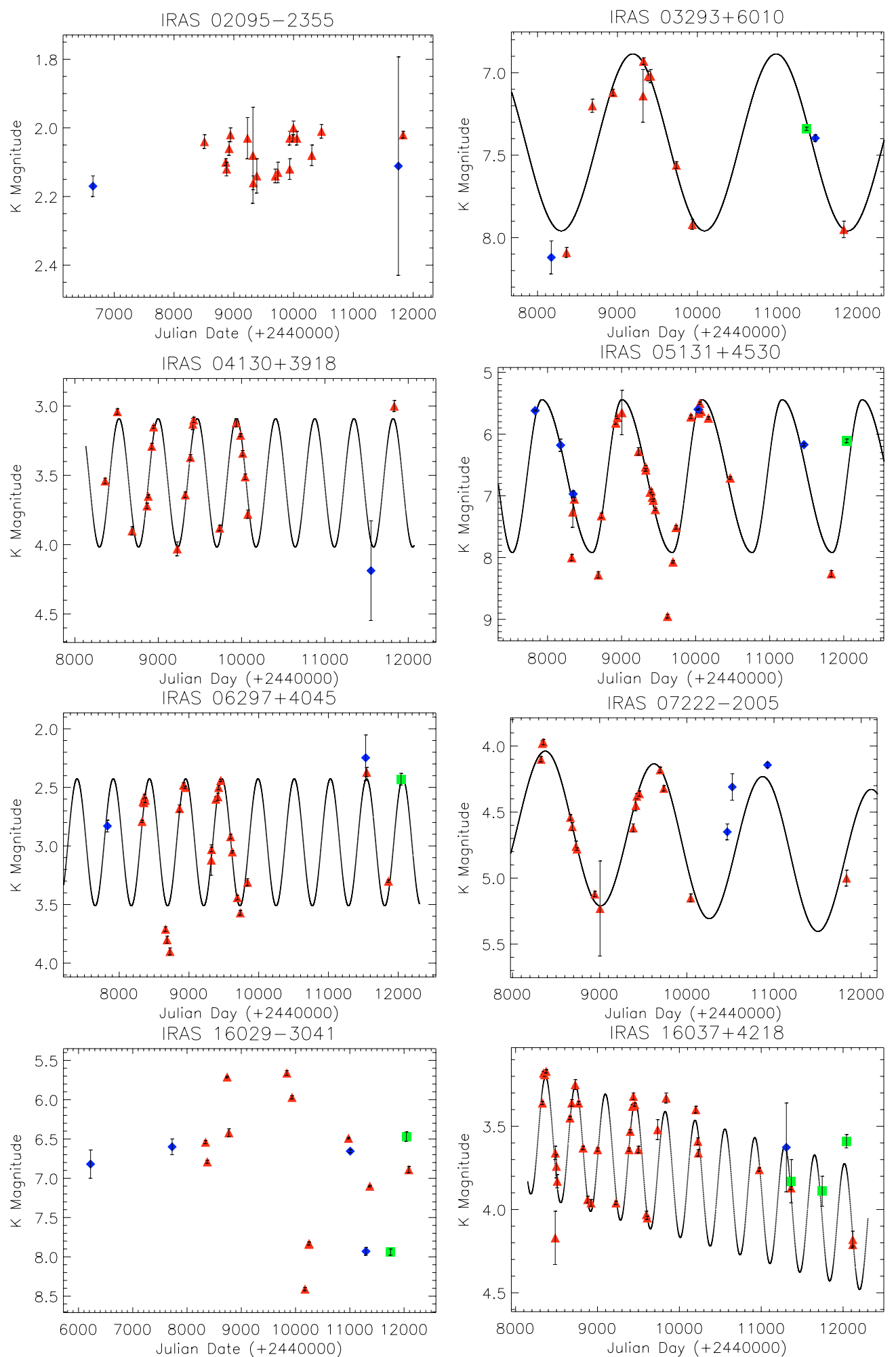

Fig. A.1. $K$-band measurements used in our variability analysis. CVF data are plotted with triangles (red), MAGIC data with squares (green), and data from literature with diamonds (blue). For IRAS 06297+4045, one data point at JD 2440510 from the literature has not been plotted. 
F. M. Jiménez-Esteban et al.: Near-IR variability properties of a selected sample of AGB stars, Online Material p 3
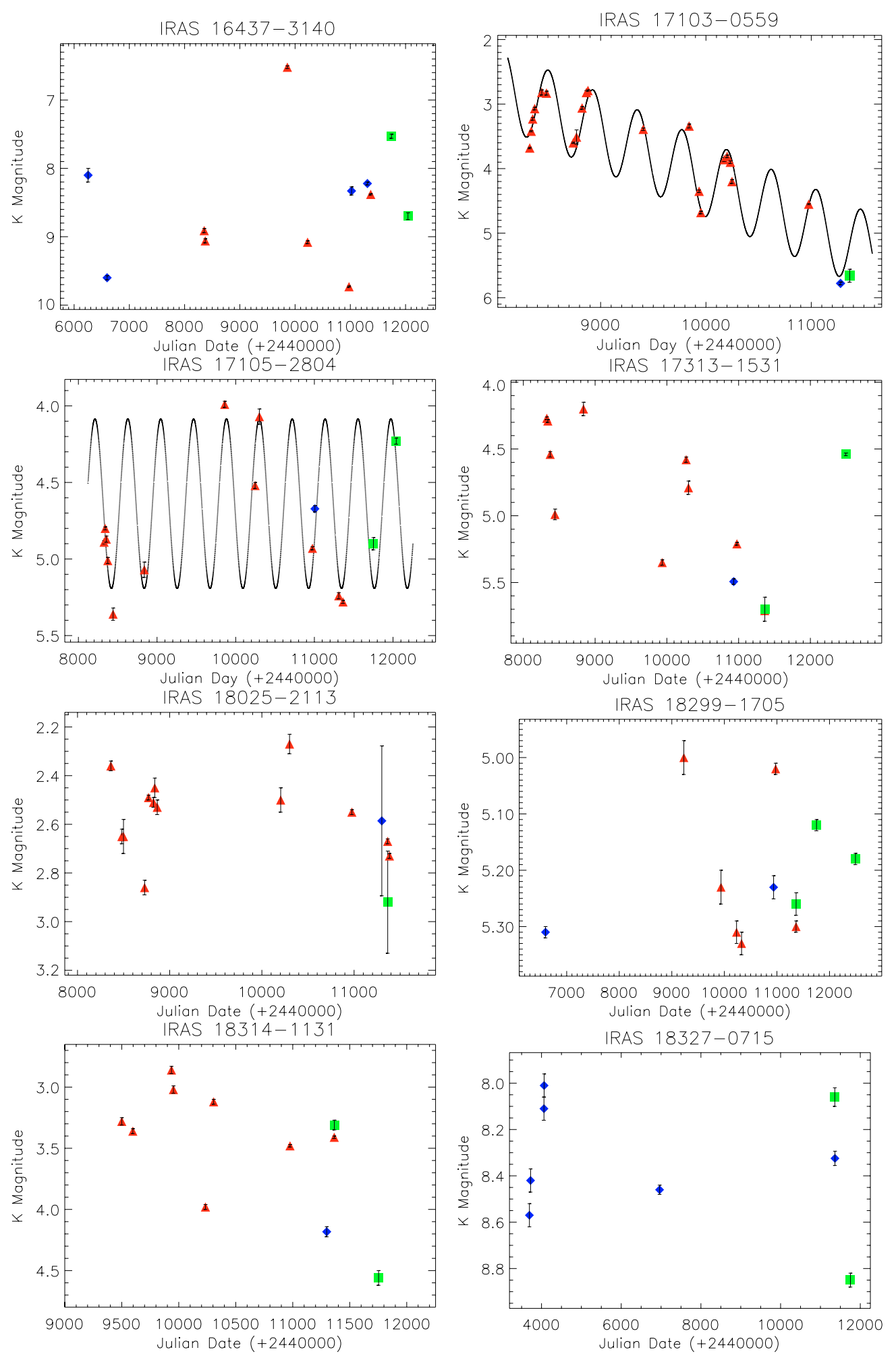

Fig. A.1. continued. 
F. M. Jiménez-Esteban et al.: Near-IR variability properties of a selected sample of AGB stars, Online Material p 4
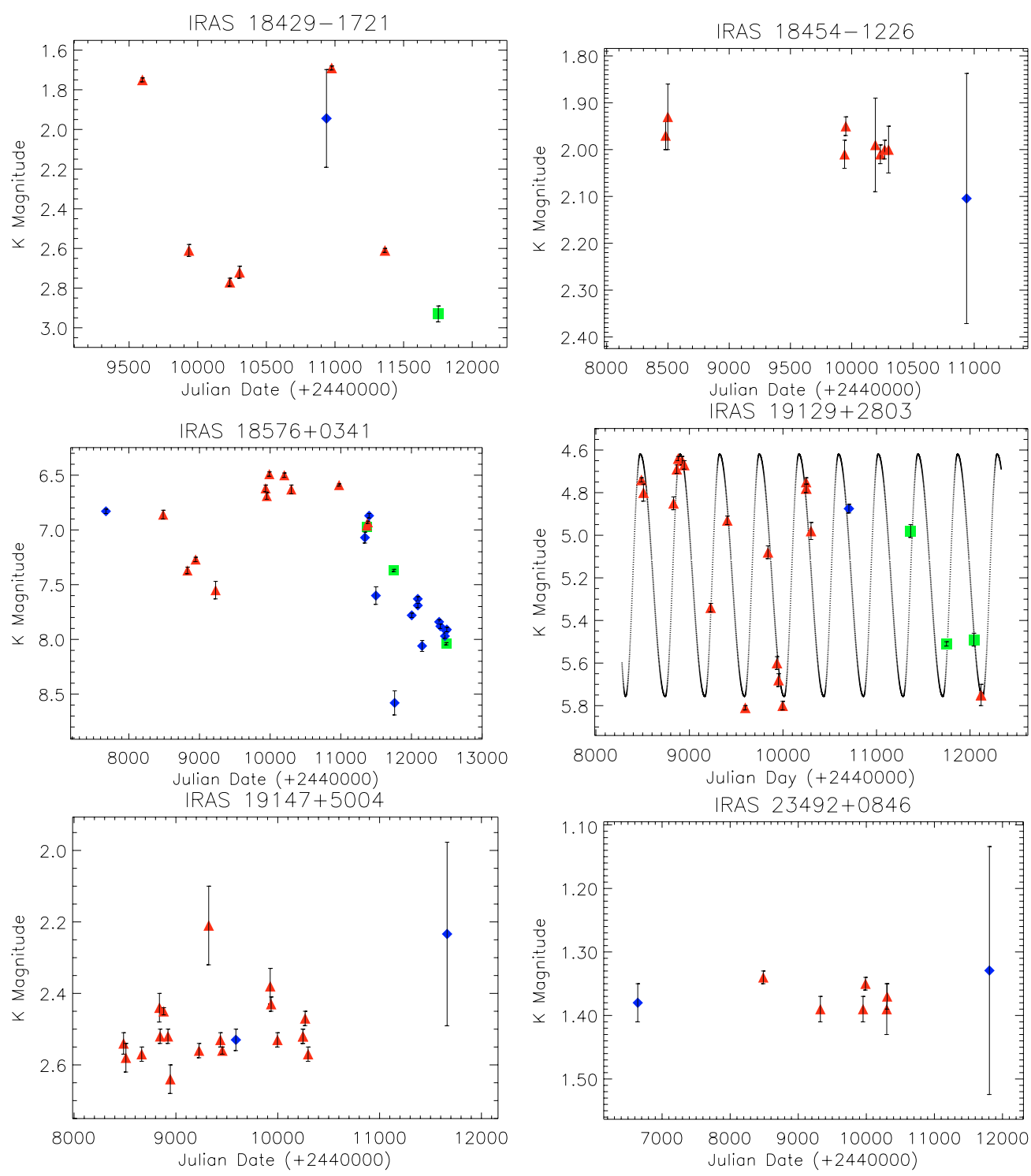

Fig. A.1. continued. 
F. M. Jiménez-Esteban et al.: Near-IR variability properties of a selected sample of AGB stars, Online Material p 5
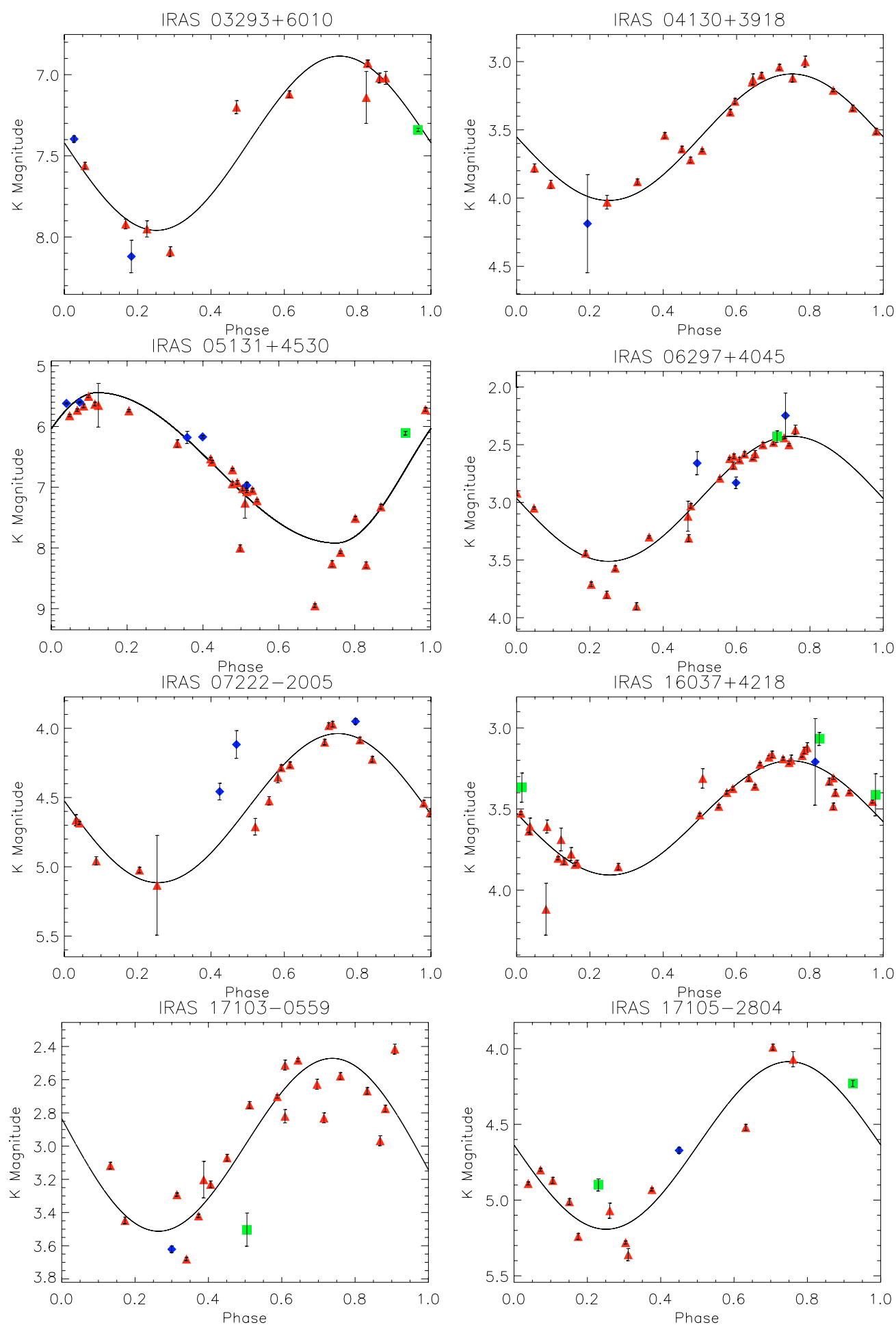

Fig. A.2. Light curves derived from the photometric data. CVF data are plotted with triangles (red), MAGIC data with squares (green), and data from the literature with diamonds (blue). 
F. M. Jiménez-Esteban et al.: Near-IR variability properties of a selected sample of AGB stars, Online Material p 6

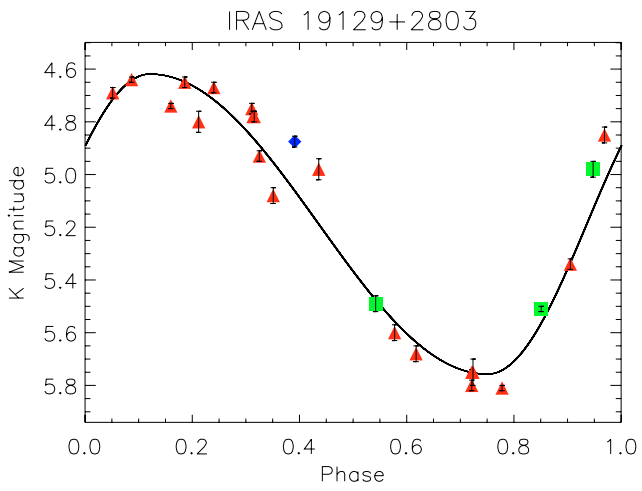

Fig. A.2. continued. 